\title{
Designing an ASIP for Cryptographic Pairings over Barreto-Naehrig Curves ${ }^{\star}$
}

\author{
David Kammler ${ }^{1}$, Diandian Zhang ${ }^{1}$, Peter Schwabe ${ }^{2}$, Hanno Scharwaechter ${ }^{1}$, \\ Markus Langenberg ${ }^{3}$, Dominik Auras ${ }^{1}$, Gerd Ascheid ${ }^{1}$, and Rudolf Mathar ${ }^{3}$ \\ 1 Institute for Integrated Signal Processing Systems (ISS), \\ RWTH Aachen University, Aachen, Germany \\ kammler@iss.rwth-aachen.de \\ 2 Department of Mathematics and Computer Science \\ Eindhoven University of Technology, Eindhoven, Netherlands \\ peter@cryptojedi.org \\ 3 Institute for Theoretical Information Technology (TI), \\ RWTH Aachen University, Aachen, Germany \\ mathar@ti.rwth-aachen.de
}

\begin{abstract}
This paper presents a design-space exploration of an application-specific instruction-set processor (ASIP) for the computation of various cryptographic pairings over Barreto-Naehrig curves (BN curves). Cryptographic pairings are based on elliptic curves over finite fields - in the case of BN curves a field $\mathbb{F}_{p}$ of large prime order $p$. Efficient arithmetic in these fields is crucial for fast computation of pairings. Moreover, computation of cryptographic pairings is much more complex than elliptic-curve cryptography (ECC) in general. Therefore, we facilitate programming of the proposed ASIP by providing a $\mathrm{C}$ compiler.

In order to speed up $\mathbb{F}_{p}$ arithmetic, a RISC core is extended with additional scalable functional units. Because the resulting speedup can be limited by the memory throughput, utilization of multiple data-memory banks is proposed.

The presented design needs $15.8 \mathrm{~ms}$ for the computation of the Optimal-Ate pairing over a 256 -bit $\mathrm{BN}$ curve at $338 \mathrm{MHz}$ implemented with a $130 \mathrm{~nm}$ standard cell library. The processor core consumes $97 \mathrm{kGates}$ making it suitable for the use in embedded systems.
\end{abstract}

Keywords: Application-specific instruction-set processor (ASIP), design-space exploration, pairing-based cryptography, Barreto-Naehrig curves, elliptic-curve cryptography (ECC), $\mathbb{F}_{p}$ arithmetic.

\footnotetext{
* This work has been supported by the UMIC Research Centre, RWTH Aachen University. The third author was supported by the European Commission through the ICT Programme under Contract ICT-2007-216499 CACE and through the ICT Programme under Contract ICT-2007-216646 ECRYPT II. Permanent ID of this document: 7e38974d56cc76a7f572f328ee4a3761. Date: 2009/06/15.
}

C. Clavier and K. Gaj (Eds.): CHES 2009, LNCS 5747, pp. 254 271, 2009.

(C) International Association for Cryptologic Research 2009 


\section{Introduction}

Pairings were first introduced to cryptography as a means to break cryptographic protocols based on the elliptic-curve discrete-logarithm problem (ECDLP) [1, [2. Joux showed in 2000 that they can also be used constructively for tripartite key agreement [3]; Subsequently, different cryptographic protocols have been presented involving cryptographic pairings, including identity-based encryption [4] and short digital signatures [5]. A discussion of various applications that would be impossible or very hard to realize without pairings is given in [6].

Cryptographic pairings are based on elliptic curves. To meet both, security requirements and computational feasibility, only elliptic curves with special properties can be considered as basis for cryptographic pairings. State-of-the-art curves for high-security applications are 256-bit Barreto-Naehrig curves (BN curves), introduced in [7]. They achieve 128-bit security according to [8] or 124-bit security according to 9]. Fast arithmetic on these curves demands for fast finite field arithmetic in a field $\mathbb{F}_{p}$ of prime order $p$, where $p$ is determined by the curve construction.

Several high-performance software implementations of pairings over BN curves exist for general-purpose desktop and server CPUs 10 11 12. However, the so far only implementation targeting an embedded system was published by Devegili et al. in [10] (updated in [13]) for a Philips HiPerSmart ${ }^{\mathrm{TM}}$ smart card; a complete pairing computation requires $5.17 \mathrm{~s}$ at $20.57 \mathrm{MHz}$, certainly too much time for interactive processes.

This result shows that in order to make state-of-the-art pairing applications available to the embedded domain we need dedicated hardware to accelerate pairing computations. However, the variety and complexity of pairing applications demand for a flexible and programmable solution, that cannot be satisfied by a static hardware implementation. Application-specific instruction-set processors (ASIPs) are a promising candidate to find a good trade-off between these contradicting demands of speed, flexibility and ease of programmability.

This paper shows a design-space exploration of an ASIP for pairing computations over BN curves. We describe how to trade off execution time against area making the ASIP suitable for use in the embedded domain. Dedicated scalable functional units are introduced that speed up general $\mathbb{F}_{p}$ arithmetic. Moreover, their critical path delay can be modified in order to be integrated with any existing RISC-like architecture without compromising its clock frequency. We show that the speedup from the special functional units is limited by a memory system with a single memory port. Hence, we introduce a memory system utilizing multiple memory banks. The number of banks can be altered without modification to the pipeline or the target architecture tools including the $\mathrm{C}$ compiler. This enables fast design-space exploration. The proposed ASIP thus offers a flexible and scalable implementation for pairing applications.

We are - up to our knowledge - the first to implement and time a complete implementation of high-security cryptographic pairings on dedicated specialized hardware. 
We would like to thank Jia Huang for supporting the implementation. We furthermore thank Daniel J. Bernstein, Tanja Lange, Ernst Martin Witte, Filippo Borlenghi, and the anonymous reviewers for suggesting many improvements to our explanations.

Related work. Several architectures for the computation of cryptographic pairings have been proposed in the literature [14, 15, 16, 17, 18, 19, 20, 21, 22, 23, 24, 25, 26. All these implementations use supersingular curves over fields of characteristic 2 or 3 , achieving only very low security levels, sometimes even below 80 bit.

Barenghi et al. recently proposed a hardware architecture for cryptographic pairings using curves defined over fields of large prime characteristic [27]. They use a supersingular curve (with embedding degree 2) defined over a 512-bit field and thus achieve only 72-bit security, according to [9].

Another architecture targeting speedup of pairings and supporting fields of large prime characteristic has been proposed in 28. The instruction set of a SPARC V8 processor is extended for acceleration of arithmetic in $\mathbb{F}_{2^{n}}, \mathbb{F}_{3^{m}}$ and $\mathbb{F}_{p}$. However, the focus is put on minor modifications of the datapath resulting in a performance gain for multiplications in $\mathbb{F}_{p}$ which is two-fold only.

The architectures closest to the one proposed in this paper are accelerating arithmetic in general $\mathbb{F}_{p}$ for elliptic-curve cryptography (ECC) 29, 30. However, these designs have not been reported to be used for pairing computations.

Some other architectures for ECC over prime fields limit their support to a prime $p$ which allows for particularly fast modular reduction (see i.e. 31]). These approaches are not adequate for pairing-based cryptography where additional properties of the elliptic curves are required. Thus, a detailed comparison with these architectures is omitted here.

Organization of the paper. Section 2 of the paper gives an overview of cryptographic pairings and Barreto-Naehrig curves. Section 3 describes our approach of an ASIP suitable for pairing computation. In Section 4 we discuss the results. The paper is concluded and future work is outlined in Section 5 .

\section{Background on Cryptographic Pairings}

We only give a short overview of the notion of cryptographic pairings, a comprehensive introduction is given in [32, chapter IX].

For three groups $G_{1}, G_{2}$ (written additively) and $G_{3}$ (written multiplicatively) of prime order $r$ a cryptographic pairing is a map $e: G_{1} \times G_{2} \rightarrow G_{3}$,

- Bilinearity: $e(k P, Q)=e(P, k Q)=e(P, Q)^{k}$ for $k \in \mathbb{Z}$.

- Non-degeneracy:

For all nonzero $P \in G_{1}$ there exists $Q \in G_{2}$ such that $e(P, Q) \neq 1$ and for all nonzero $Q \in G_{2}$ there exists $P \in G_{1}$ such that $e(P, Q) \neq 1$.

- Computability:

There exists an efficient algorithm to compute $e(P, Q)$ given $P$ and $Q$. 
We consider the following construction of cryptographic pairings: Let $E$ be an elliptic curve defined over a finite field $\mathbb{F}_{p}$ of prime order. Let $r$ be a prime dividing the group order $\# E\left(\mathbb{F}_{p}\right)=n$ and let $k$ be the smallest integer, such that $r \mid p^{k}-1$. We call $k$ the embedding degree of $E$ with respect to $r$. Let $t$ denote the trace of Frobenius fulfilling the equation $n=p+1-t$.

Let $P_{0} \in E\left(\mathbb{F}_{p}\right)$ and $Q_{0} \in E\left(\mathbb{F}_{p^{k}}\right)$ be points of order $r$ such that $Q_{0} \notin\left\langle P_{0}\right\rangle$, let $\mathcal{O} \in E\left(\mathbb{F}_{p}\right)$ denote the point at infinity. Define $G_{1}=\left\langle P_{0}\right\rangle$ and $G_{2}=\left\langle Q_{0}\right\rangle$. Let $G_{3}=\mu_{r}$ be the group of $r$-th roots of unity in $\mathbb{F}_{p^{k}}^{*}$.

For $i \in \mathbb{Z}$ and $P \in E$ a Miller function 33 is an element $f_{i, P}$ of the function field of $E$, such that the principal divisor of $f_{i, P}$ is $\operatorname{div}\left(f_{i, P}\right)=i(P)-([i] P)-(i-1) \mathcal{O}$.

Using such Miller functions, we can define the map

$$
e_{s}: G_{1} \times G_{2} \rightarrow \mu_{r} ;(P, Q) \mapsto f_{s, P}(Q)^{\left(p^{k}-1\right) / r} .
$$

For certain choices of $s$ the map $e_{s}$ is non-degenerate and bilinear. For $s=r$ we obtain the reduced-Tate pairing $\tau$ and for $s=T=t-1$ we obtain the reducedAte pairing $\alpha$ by switching the arguments 34. Building on work presented in 35, Vercauteren introduced the Optimal-Ate pairing in 36] which for BN curves can be computed using $s \approx \sqrt{t}$ and a few additional computations (see also [37]).

Using twists of elliptic curves we can further define the generalized reduced- $\eta$ pairing [34, 38. In [12 a method to compute the Tate and $\eta$ pairing keeping intermediate results in compressed form is introduced. We refer to the resulting algorithms as Compressed-Tate and Compressed- $\eta$ pairing, respectively.

\subsection{Choice of an Elliptic Curve}

For cryptographic protocols to be secure on the one hand and the pairing computation to be computationally feasible on the other hand, the elliptic curve $E$ must have certain properties: Security of cryptographic protocols based on pairings relies on the hardness of the discrete logarithm problem in $G_{1}, G_{2}$ and $G_{3}$. For the 128-bit security level, the National Institute of Standards and Technology (NIST) recommends a prime group order of 256 bit for $E\left(\mathbb{F}_{p}\right)$ and of 3072 bit for the finite field $\mathbb{F}_{p^{k}}$ [8].

Barreto-Naehrig curves, introduced in [7], are elliptic curves over fields of prime order $p$ with embedding degree $k=12$. The group order $n=r$ of $E\left(\mathbb{F}_{p}\right)$ is prime by construction, the values $p$ and $n$ can be given as polynomial expressions in an integer $u$ as follows:

$$
\begin{aligned}
& p=p(u)=36 u^{4}+36 u^{3}+24 u^{2}+6 u+1 \text { and } \\
& n=n(u)=36 u^{4}+36 u^{3}+18 u^{2}+6 u+1 .
\end{aligned}
$$

For our implementation we follow [10] and set $u=0 \times 6000000000001 \mathrm{~F} 2 \mathrm{D}$, yielding two primes $p(u)$ and $n(u)$ of $l=256 \mathrm{bit}$. The field size of $\mathbb{F}_{p^{k}}$ then has $256 \cdot k=3072$ bit. Note, that according to [9], a finite fields of size 3072 bit offers only 124-bit security. In this paper we follow the more conservative estimations of [9] and claim only 124-bit security for pairings over 256-bit BN curves. 


\subsection{Computation of Pairings}

The computation of cryptographic pairings consists of two main steps: the computation of $f_{s, P}(Q)$ for Tate and $\eta$ pairings or of $f_{s, Q}(P)$ when considering the Ate pairing and the final exponentiation with $\left(p^{k}-1\right) / r$.

The first part is usually done iteratively using variants of Miller's algorithm 33. Several optimizations of this algorithm have been presented in [39. The resulting algorithm is often referred to as BKLS algorithm. For BN curves even more optimizations can be applied by exploiting the fact that such curves have sextic twists. A detailed description of efficient computation of pairings over BN curves, including the computation of Miller functions and the final exponentiation is given in [10]. Our implementation follows this description in large parts.

Finite field computations constitute the bulk of the pairing computation in software implementations typically more than $90 \%$ of the time is spent on modular multiplication, inversion and addition, the number of these operations for the implemented pairing algorithms is the following:

\begin{tabular}{lrrrrrr}
\hline Number of & Opt. Ate & \multicolumn{1}{c}{ Ate } & \multicolumn{1}{c}{$\eta$} & Tate & Comp. $\eta$ & Comp. Tate \\
\hline multiplications & 17,913 & 25,870 & 32,155 & 39,764 & 75,568 & 94,693 \\
additions & 84,956 & 121,168 & 142,772 & 174,974 & 155,234 & 193,496 \\
inversions & 3 & 2 & 2 & 2 & 0 & 0 \\
\hline
\end{tabular}

Throughout the pairing computation we keep points on elliptic curves in Jacobian coordinates and can thus almost entirely avoid field inversions; our targets for hardware acceleration are thus multiplication and addition in $\mathbb{F}_{p}$, inversion is implemented as exponentiation with $p-2$.

\section{An ASIP for Cryptographic Pairings}

To implement various pairing algorithms (Optimal Ate, Ate, $\eta$, Tate, Compressed $\eta$ and Compressed Tate), a programmable and therefore flexible architecture is targeted in this paper. Standard architectures like embedded RISC cores are flexible, but they are lacking sufficient computational performance for specific applications. Therefore, we apply the ASIP concept to cryptographic-pairing applications in order to reduce the computation time while maintaining programmability. Development and implementation of our ASIP have been carried out using the Processor Designer from CoWare [40].

Keeping control over the data flow on the higher layers of the pairing computation, like $\mathbb{F}_{p^{12}}$ or $E\left(\mathbb{F}_{p^{2}}\right)$ arithmetic, is a rather complex task. This calls for a convenient programming model. However, on the lower level realizing the $\mathbb{F}_{p}$ arithmetic, computational performance is of highest priority. Therefore, we decided to extend a basic 5-stage 32-bit RISC core with special $\mathbb{F}_{p}$ instructions for modular multiplication, addition and subtraction. Inversions are not considered for special instructions as they are used very seldom $(\leq 3 \times)$ in any of 
the targeted applications. The available $\mathrm{C}$ compiler enables convenient application development on higher levels, while the computational intensive tasks are mapped to the specialized instructions accessible via intrinsic: 1 .

Among the targeted $\mathbb{F}_{p}$ operations, the most challenging one to implement is fast modular multiplication, especially for a large word width (e.g. 256 bit). In general, multiplication in $\mathbb{F}_{p}$ can be done by first multiplying the two 256-bit factors and then reducing the 512-bit product. This might indeed be the fastest approach, if $p$ could be chosen of a special form as for example specified in [4] or [42. However, due to the construction of BN curves (see [7]) we cannot use such primes. Therefore, our approach uses Montgomery arithmetic [43].

\subsection{Data Processing: A Scalable Montgomery-Multiplier Unit}

In 1985 Montgomery introduced an algorithm for modular multiplication of two integers $A$ and $B$ modulo an integer $M$ [43. The idea of the algorithm is to represent $A$ as $\hat{A}=A R \bmod M$ and $B$ as $\hat{B}=B R \bmod M$ for a fixed integer $R>M$ with $\operatorname{gcd}(R, M)=1$. This representation is called Montgomery representation. To multiply two numbers in Montgomery representation we have to compute $\widehat{A B}=\hat{A} \hat{B} R^{-1} \bmod M$. For certain choices of $R$ this computation can be carried out much more efficiently than usual modular multiplication: Let us assume that $M$ is odd and let $l$ be the bit length of $M$. Choosing $R=2^{l}$ clearly fulfills the requirements on $R$ and allows for modular multiplication that replaces division operations by shifts, allowing for an efficient hardware implementation.

In the context of $\mathbb{F}_{p}$-multiplication the modulus $M$ corresponds to $p$. All $\mathbb{F}_{p}$ operations can be performed in Montgomery representation. Therefore, all values can be kept in Montgomery representation throughout the whole pairing computation.

Nibouche et al. introduced a modified version of the Montgomery multiplication algorithm in 44. It splits the algorithm into two multiplication operations, that can be carried out simultaneously, and allows for using carry save (CS) multipliers. This results in a fast architecture that can be pipelined and segmented easily. Therefore, it is chosen as basis for our development. A $4 \times 4$-bit example is shown in Fig. 1 .

The actual multiplication is carried out in the left half of the architecture, while the reduction is performed in the right part simultaneously. The left part is a conventional multiplier built of gated full adders (gFAs), whereas the right part consists of a multiplier with special cells for the least-significant bits (LSBs). The LSB cells are built around a half adder (HA). Their overall delay is comparable to that of a gFA. A more detailed description can be found in [44].

Due to area constraints we decided to implement only subsets of the regular structures of the multiplier and perform the computation in multiple cycles. The CS-based design provides the opportunity to not only make horizontal but also vertical cuts while the critical path of the multiplier unit depends on its height

${ }^{1}$ An adoption of our code to general purpose processors using the GMP library instead of intrinsics is available from http://cryptojedi.org/crypto/ 


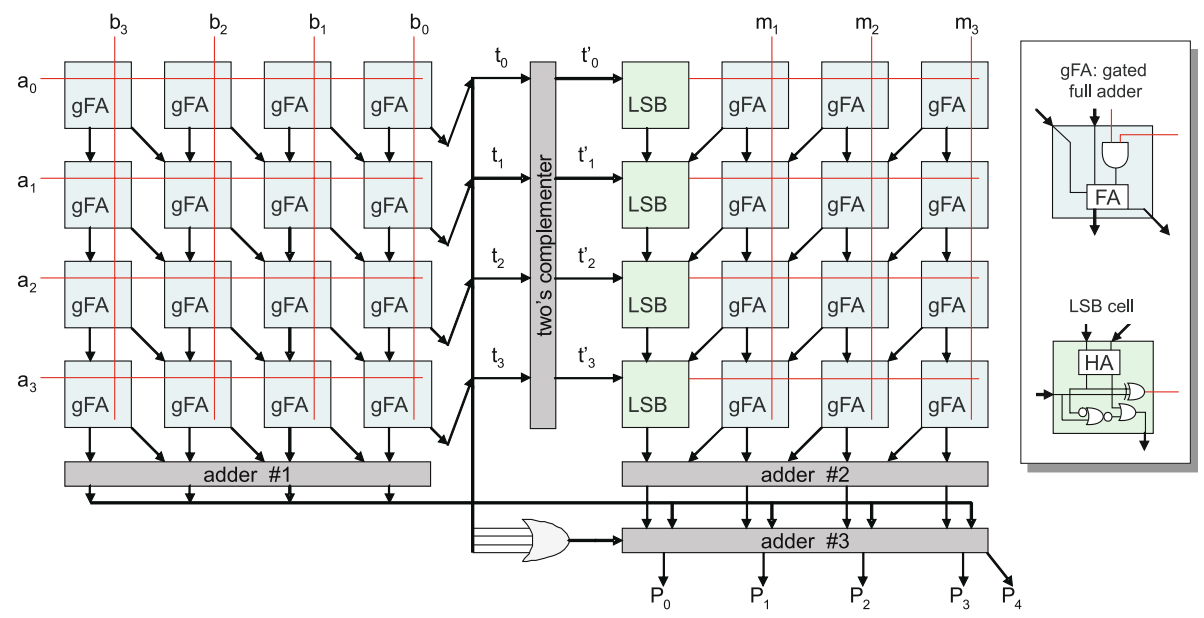

Fig. 1. Montgomery-multiplier based on Nibouche et al. [44]

$(H)$ only. This makes the design adaptable to existing cores in terms of timing maintaining the performance of their general instruction set. Once the height of the multiplier unit is chosen (in our case $H=8$ ), the width $(W)$ can be selected to adapt the design to the desired computational performance and to trade off area vs. execution time of the multiplication.

Multiplication and reduction are carried out simultaneously starting from the most-significant bit (MSB) of their second operand ( $B$ and $M$ ) first. However, the reduction cannot be started until the incoming data for the LSB cells are available from the two's complementer. Therefore, reduction starts after the first $H$ lines of multiplication have been executed and remains delayed for $\left\lceil\frac{l}{W}\right\rceil$ cycles (required for the computation of $H$ lines). Eventually, the CS results need to be transformed back to two's complement number representation (by addition \#1 and addition \#2) before they are combined to the result by addition \#3. This is necessary since the result lies in the range of 0 to $2 M-1$, and requires a final comparison against $M$, which is difficult to handle in CS representation. The comparison including a necessary subtraction of $M$ is performed in another functional unit introduced later. Equation (1) gives the number of required cycles $c_{M M}$ to perform a Montgomery multiplication with the proposed multi-cycle architecture for the general case.

$$
c_{M M}=\left(\left\lceil\frac{l}{H}\right\rceil+1\right) \cdot\left\lceil\frac{l}{W}\right\rceil+2
$$

For evaluation, we implemented this multi-cycle Montgomery-multiplier (MMM) in three different sizes $(W \times H): 32 \times 8$ bit, $64 \times 8$ bit and $128 \times 8$ bit, resulting in an execution time of 266, 134 and 68 cycles respectively. However, the area savings for smaller (and slower) architectures do not scale as well as the execution time. This results from the increased complexity of the required multiplexing for smaller MMM units. In order to keep the amount of multiplexers small, we 


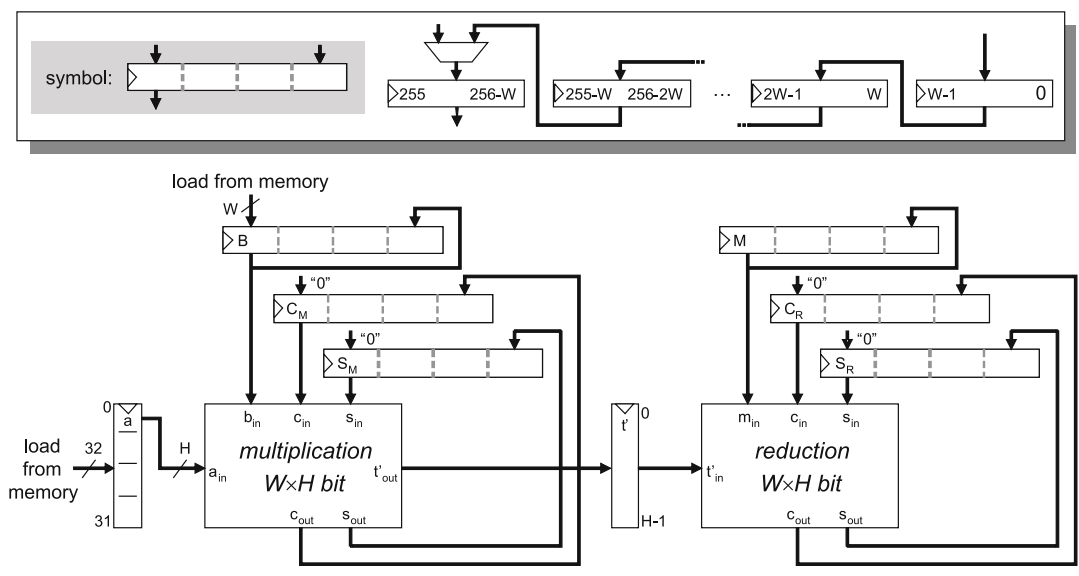

Fig. 2. Structure of the multi-cycle Montgomery-multiplier (MMM)

designed special 256-bit shift registers, that enable a circular shift by $W$ bits for the operands $B, M$ and the corresponding intermediate CS values. This solution is suitable, since the input values are accessed in consecutive order by blocks of $W$ bits. Still, area savings when scaling a $128 \times 8$-bit architecture down to $32 \times 8$-bit are about $50 \%$.

Fig. 2 shows the overall resulting structure of the MMM unit. The two's complementer is included in the multiplication unit, while the reduction unit contains additional LSB cells that produce input for the gFA cells on the fly (as depicted in Fig. (1). The input shift registers are initialized step by step during the first $\left\lceil\frac{l}{W}\right\rceil$ cycles. After the whole process, the result is stored in the registers for temporary CS values $\left(C_{M}, S_{M}, C_{R}, S_{R}\right)$. The adders for the final summations are not depicted.

An advantage of stepwise executing the multiplication is that the total multiplication width $l$ can be configured at runtime in steps of $W$. The overall dependence of the execution time on $l$ is quadratic. Modular multiplication is thus significantly faster for smaller multiplication width. This may be interesting for ECC applications requiring lower security.

Similar to the MMM unit we developed a multi-cycle adder unit for modular additions and subtractions, which reads two operands block-wise and simultaneously. For evaluation, a 32-bit and a 64-bit version of this unit have been implemented. Details are omitted here since the implementation is straightforward.

Both, MMM and adder unit require a final subtraction of $M$ whenever the result exceeds this prime number. A special writeback unit takes care of this subtraction right before writing back the data, operating block-wise in multiple cycles as well. This unit has been implemented with a width of 32,64 and 128 bit.

During the execution of multi-cycle operations for modular addition, subtraction and multiplication the pipeline is stalled. Three special instructions are implemented triggering these operations. Instruction arguments are registers containing the starting address of each of the three 256-bit operands. Since 
the modulus $M$ is not changed during an application run, a special register is utilized and implicitly accessed by the instructions. This register is initialized with $p$ at the beginning of an application via another dedicated instruction.

\subsection{Data Access: An Enhanced Memory Architecture}

Due to the large width of the operands, the existing 16x32-bit general purpose register file could only store two operands simultaneously. This results in frequent memory accesses consuming additional cycles and thus decreasing the overall performance of the architecture significantly. Enlarging the register file would be very costly in terms of area consumption. Hence, the instructions triggering the multi-cycle operations for modular addition, subtraction and multiplication are implemented as memory-to-memory instructions. This way, the memory accesses can be almost completely hidden in the actual computation.

The resulting throughput demands on the memory system are quite high. Especially the modular addition/subtraction requires a throughput higher than one 32-bit word per cycle. The following two evident mechanisms to increase memory throughput for ASIP designs are not well suited here: First, using memories with multiple ports is costly. The number of ports is limited to two for SSRAMs and the required area is roughly doubled. Second, designing a dedicated system with several (often specialized) memories targets highest performance, but is a complex task. The data memory space gets segmented irregularly, making it difficult to access and manage for a compiler.

Due to the drawbacks of these two approaches we apply a different technique, which we would like to introduce as transparent interleaved memory segmentation (TIMS). Its basic principle is to extend the number of ports to the memory system in order to increase the throughput by using memory banks. These banks are selected on the basis of address bits and can be accessed in parallel. In case of our ASIP, the LSBs of the address are used for the memory bank selection. This results in an addressing scheme, where the memory is selected by calculating the address modulo the number of memories $m_{d}$, which has to be a power of two.

In principle, the distribution of accesses to a banked memory system can be handled in software or hardware. However, memory-access conflicts can occur when simultaneous accesses refer to the same memory. Solving these conflicts in software requires an extension of the $\mathrm{C}$ compiler in order to avoid multiple simultaneous accesses to the same memory bank. If these conflicts can be ruled out at compile time, this approach results in very efficient code. However, if the conflicts do occur at runtime, additional code to resolve the conflict needs to be included in the target software at the cost of increased execution time. Especially when pointers are used and function calls have a substantial degree of nesting (which is the case for the targeted pairing applications), detecting the conflicts at compile time is often impossible. It requires a significant extension of the $\mathrm{C}$ compiler functionality and comes at the cost of increased code size and execution time.

However, due to fairly simple mechanisms and regularity, the distribution of accesses to the memories and resolution of access conflicts can be handled efficiently at runtime by a dedicated hardware block, the memory-access unit 
(MAU) that distributes the memory accesses from the pipeline to the correct memory. Memory accesses are requested concurrently by the pipeline on demand resulting in multiple independent read or write connections (unidirectional) between pipeline and MAU. The MAU takes care of granting accesses. Therefore, a simple handshaking protocol is used between pipeline and MAU, which is able to confirm a request within the same cycle in order not to cause any delay cycles when trying to access the fast SSRAMs.

One advantage of this mechanism is, that from the perspective of the core, the memory space remains unchanged, regardless of the number of memories. Existing load and store instructions are sufficient to access the whole data memory space. Even when special instructions perform concurrent memory accesses, a modification in the memory system (e.g. changing number of memories) does not result in a change of the core or the $\mathrm{C}$ compiler. This enables orthogonal implementation and modification of the base architecture and the memory system.

A priority-based hardware resolution of access conflicts is implemented in the MAU in two ways. Static priorities can be used if certain accesses always have higher priority than others. For instance write accesses from later pipeline stages should always have higher priority than read accesses from prior stages. When the priority is changing at runtime, dynamic priority management is required. Then, dedicated additional communication lines between core and MAU indicate a change of priority. In our design this is required by the adder unit.

Fig. 3 depicts the four different connection schemes between MAU and pipeline. The number and type of connections between MAU and pipeline are determined by the number and type of independent memory accesses initiated by the pipeline, while the number of actual memory connections depends on the number of attached data memories $m_{d}\left(m_{d}=2\right.$ in this example). For sake of clarity, the actual interconnections within the MAU have been omitted in Fig. 3. The access-control block combines the enable and priority signals with the $\log _{2}\left(m_{d}\right)$ LSBs of the address signals from the read and write connections in order to produce the grants. At the same time the enable signals for the SSRAMs are set accordingly by this unit. It also controls the crossbars that are switching the correct address, read data and write data signals to the memory ports. Please note, that the read data crossbar is switched with one cycle delay compared to the address crossbar in order to be in sync with the single cycle read latency of the SSRAMs. Effects of TIMS on physical parameters like timing and area consumption are discussed in detail in the result section.

Overall, the TIMS approach enables to extend any existing architecture with memory banks without altering the existing pipeline or the basic instruction set. The specialized memory-to-memory instructions can take full advantage of parallel accesses to these banks reducing execution time. It is not necessary to extend the $\mathrm{C}$ compiler of the base architecture with more than intrinsics for the special instructions. The compiled executable can be used on any architecture variant independently from the number of memory banks. As the MAU hides the actual memory system from the pipeline, the number of memory banks can be changed without modification to the pipeline enabling a fast design space exploration. 


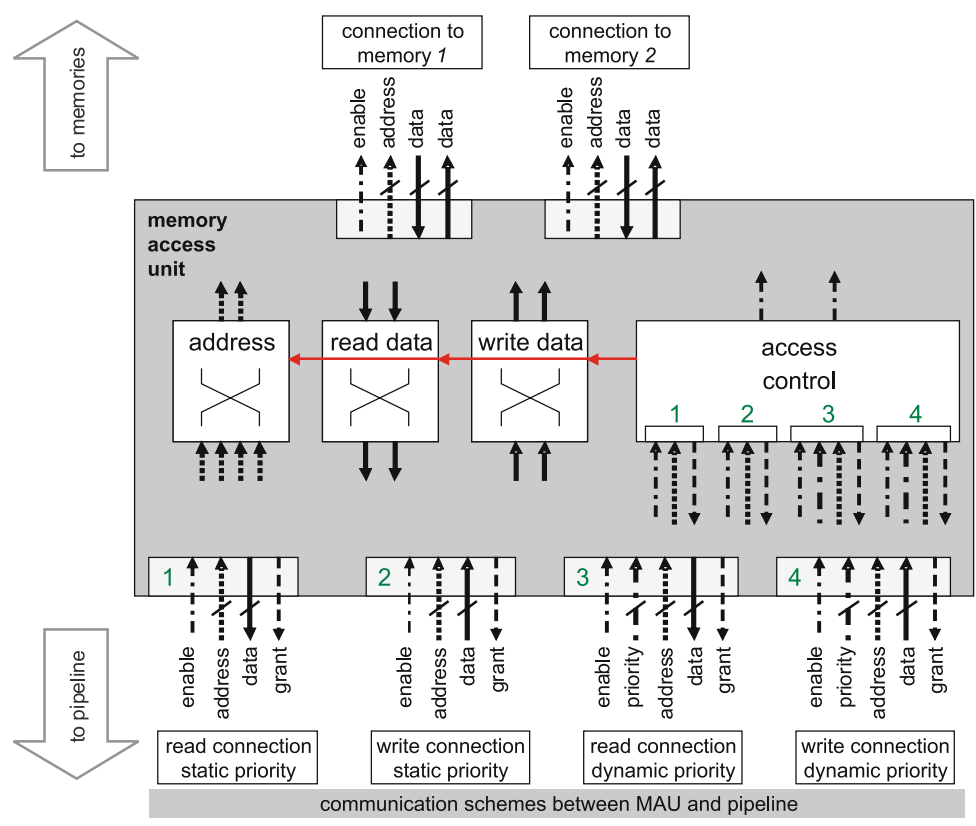

Fig. 3. Interconnect of memory-access unit (MAU)

Still, memory access collisions decrease the performance of the system and cannot be avoided completely due to the automatic address management of the $\mathrm{C}$ compiler. However, in our case this effect is kept minimal due to the good distribution of the 256-bit words. For additions and multiplications this causes a maximum additional delay of one cycle only. This results in a maximum performance degradation caused by memory-access conflicts of less than $2 \%$ for any of the implemented pairing applications.

\section{Results}

Overall, we have implemented nine variants of our ASIP with different design parameters regarding number of data memories and width of the computational units for modular multiplication, modular addition and multi-cycle writeback (Table 1). The number of data memories is closely coupled with the width of the adder and the writeback unit. Other combinations would operate functionally correctly, but would waste either performance or area. The implementation of a 16-bit adder for the single memory case would not significantly reduce area due to additional multiplexing and is therefore neglected. All synthesis results have been obtained with Synopsys Design Compiler 45 using a $130 \mathrm{~nm}$ CMOS standard cell library with a supply voltage of $1.2 \mathrm{~V}$ and are given before place and route. The memories are synchronous single-port SRAMs with a latency of one cycle. The total data-memory size is 2048 words for each of the design 
Table 1. Implemented design variants of the ASIP for pairings

\begin{tabular}{|c|c|c|c|c|c|c|c|c|c|}
\hline Variant & $128 m_{4}$ & $64 m 4$ & $32 m_{4}$ & $128 \mathrm{~m}^{2}$ & $64 m_{2}$ & $32 m_{2}$ & $128 m 1$ & $64 m 1$ & $32 m 1$ \\
\hline ll size (bit) & $128 \times 8$ & $64 \times 8$ & $32 \times 8$ & $128 \times 8$ & $64 \times 8$ & $32 \times 8$ & $128 \times 8$ & $64 \times 8$ & $32 \times 8$ \\
\hline mod add width (bit) & 64 & 64 & 64 & 32 & 32 & 32 & 32 & 32 & 32 \\
\hline writeback width (bit) & 128 & 128 & 128 & 64 & 64 & 64 & 32 & 32 & 32 \\
\hline \# data memories & 4 & 4 & 4 & 2 & 2 & 2 & 1 & 1 & 1 \\
\hline total area $^{a}$ (kGates) & 195 & 186 & 182 & 164 & 153 & 148 & 145 & 134 & 130 \\
\hline core area ${ }^{b}$ (kGates) & 96 & 87 & 83 & 97 & 86 & 81 & 93 & 83 & 79 \\
\hline timing (ns) & 3.69 & 3.65 & 3.52 & 2.96 & 2.97 & 3.02 & 2.95 & 3.03 & 3.09 \\
\hline Optin & 7.5 & 21.8 & 29.9 & 8 & 19.4 & 27.3 & 9.2 & 23.4 & 32.0 \\
\hline Ate $(m s)$ & 25.3 & 31.4 & 42.6 & 22.8 & 27.9 & 38.9 & 27.6 & 33.5 & 45.6 \\
\hline$\eta(m s)$ & 32.3 & 39.5 & 52.8 & 28.8 & 35.0 & 48.1 & 34.6 & 41.6 & 56.2 \\
\hline Tate (ms) & 38.5 & 47.0 & 62.7 & 34.4 & 41.6 & 57.1 & 41.1 & 49.5 & 65.3 \\
\hline Compressed $\eta(m s)$ & 38.6 & 55.0 & 86.2 & 34.5 & 48.2 & 77.1 & 41.6 & 56.5 & 85.8 \\
\hline Compressed Tate (ms) & 48.2 & 68.9 & 107.8 & 43.2 & 60.3 & 96.5 & 52.0 & 70.7 & 107.3 \\
\hline
\end{tabular}

${ }^{a}$ Including area for data memories.

${ }^{b}$ Without area for memories, but including area for MAU.

variants. The program memory is not included in the area reports, since it is not changing through the different designs and could be implemented differently (as ROM, RAM, synthesized logic etc.) depending on the final target system. The plain RISC (32-bit, 5-stage pipeline, 32-bit integer multiplier) without memories and extensions consumes 26 kGates and achieves a timing of $2.89 \mathrm{~ns}$.

Fig. 4 shows the area distribution of the different ASIP variants. While the basic core only shows moderate area increase from 17 to $21 \mathrm{kGates}$ for all

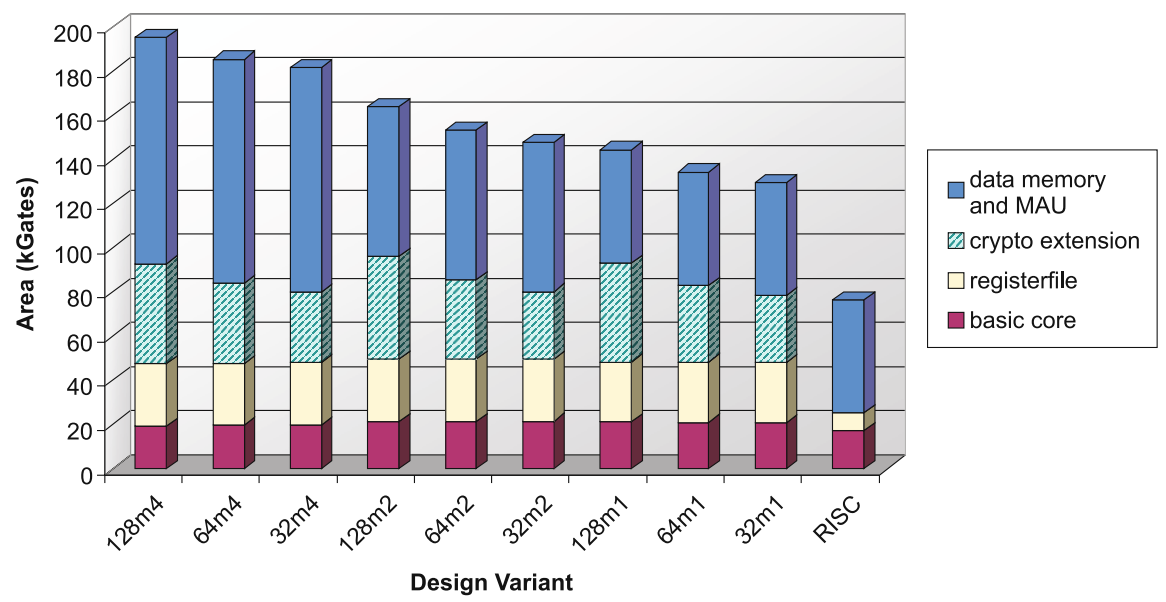

Fig. 4. ASIP area consumption and distribution 


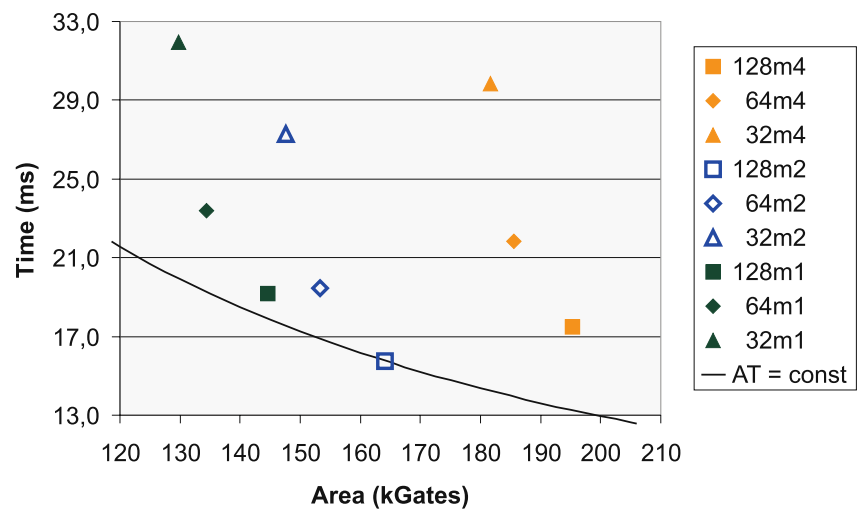

Fig. 5. Area-time trade-off for different ASIP variants (Optimal Ate pairing)

variants (resulting from decoder extensions and additional pipeline registers), the area for the register file increases from 9 to 28 kGates compared to the plain RISC. The reason are specialized 256-bit registers storing the prime number and intermediate results of the modular operations. These registers are independent from the width of any of the additional functional units. The area of the cryptographic extensions is dominated by the MMM unit.

Observe that splitting the memory into two of half the size results in a datamemory area increase of $31 \%$. Utilizing a dual port memory instead would increase area by over $83 \%$. The area overhead due to the MAU lies between only 0.5 and 1.2 kGates, when two memories are attached. Even for four attached memories it is below $3.5 \mathrm{kGates}$.

However, limitations of TIMS utilizing the proposed MAU become visible when looking at the timing of the different variants of the ASIP. While attaching one or two data memories barely affects the critical path with respect to the original RISC architecture (within design tool accuracy, see Table 1), an increased delay is observed when four memories are attached. This delay is caused by the complexity of priority resolution for four attached memories combined with four independent memory accesses with dynamic priority, which are necessary to implement the 64-bit adder.

The execution times of all six implemented pairing applications on all nine ASIP variants are shown in Table 1] For all applications performance improves significantly with increasing width of the MMM. Also, the number of cycles decreases when increasing the number of connected data memories. Unfortunately, the longer critical path of the four-memory system leads to a lower performance than for the designs with two memories. The overall fastest design is variant 128m2, executing the Optimal-Ate pairing in $15.8 \mathrm{~ms}$. With the smallest and slowest variant completing the task in $32.0 \mathrm{~ms}$, the user is offered a quite broad design space enabling trade-offs.

In order to evaluate the efficiency of the different design variants, Fig. 5 shows the area-time trade-off for the Optimal-Ate pairing. It can be seen clearly that the best AT product is obtained by the $128 \mathrm{~m} 2$ design. This shows the importance of 
investigating the memory architecture of ASIPs during design-space exploration. In our case the best results are obtained with TIMS and two data memories in spite of the considerable area increase due to the memory splitting.

\subsection{Performance Comparison}

To our best knowledge there exists no literature reporting performance figures resulting from actual implementations of cryptographic pairings on dedicated hardware achieving a 124-bit security level. Hardware implementations for lower security levels can obviously be much faster than the proposed design.

Table 2 gives an overview of performance and area consumption for various pairing implementations on dedicated hardware; the given security levels are according to 9 . Whenever more than one design variant is given in a publication, the fastest one with the highest security level is listed in the table. The previous results listed in Table 2 are hardly comparable to the design proposed in this paper. Not only do they achieve lower security levels, they also mainly focus on FPGAs rather than standard cells and mostly use curves over binary or ternary fields. In the following we therefore give a comparison with standard cell designs which accelerate $\mathbb{F}_{p}$ arithmetic for elliptic-curve cryptography and finally discuss our design in the context of smart cards.

Comparison with standard cell designs for ECC. Other publications describing dedicated-hardware implementations for ECC over fields of large prime

Table 2. Performance and area comparison for pairings

\begin{tabular}{|c|c|c|c|c|c|c|c|}
\hline Design & Technology & Area & $\begin{array}{l}\text { Freq. } \\
(\mathrm{MHz})\end{array}$ & Pairing & $\begin{array}{c}\text { Field } \\
\text { Characteristic }\end{array}$ & $\begin{array}{c}\text { Secu- } \\
\text { rity } \\
\text { (bit) }\end{array}$ & $\begin{array}{l}\text { Time } \\
\text { (ms) }\end{array}$ \\
\hline this work & $130 \mathrm{~nm}$ std. cell & 97 kGates & 338 & Opt. Ate & 256-bit prime & 124 & 15.8 \\
\hline 14 & Xilinx xc2vp20 & 8 kSlices & 90 & Tate & 3 & 97 & 0.298 \\
\hline 14 & Xilinx xc2vp20 & 8 kSlices & 115 & Tate & 2 & 97 & 0.327 \\
\hline 21 & Xilinx xc2vp100 & 44 kSlices & 33 & Tate & 2 & 80 & 0.146 \\
\hline 18, 46 & Xilinx xc2vp100 & 38 kSlices & 72 & Tate & 2 & 76 & 0.049 \\
\hline 20 & Xilinx xc2v6000 & 25 kSlices & 47 & Tate & 2 & 76 & 2.81 \\
\hline 19 & Xilinx xc2v6000 & 15 kSlices & 40 & Tate & 2 & 76 & 3.0 \\
\hline 27 & Xilinx xc2v8000 & 34 kSlices & 135 & Tate & 512-bit prime & 72 & 1.61 \\
\hline 23 & Xilinx xc4vlx200 & 74 kSlices & 199 & $\eta_{T}$ & 3 & 68 & 0.008 \\
\hline 16 & Altera ep2c35 & $19 \mathrm{kLEs}$ & 147 & $\eta_{T}$ & 3 & 68 & 0.027 \\
\hline 17 & $180 \mathrm{~nm}$ std. cell & 194 kGates & 200 & $\eta_{T}$ & 3 & 68 & 0.047 \\
\hline 15 & Xilinx xc4vlx15 & $2 \mathrm{kSlices}$ & 203 & $\eta_{T}$ & 3 & 68 & 0.137 \\
\hline 25 & Xilinx xc2vp100 & 15 kSlices & 85 & $\eta_{T}$ & 3 & 68 & 0.183 \\
\hline 26 & Xilinx xc2vp200 & 14 kSlices & 77 & Tate & 3 & 68 & 0.251 \\
\hline 22 & Xilinx xc2vp4 & 4 kSlices & 150 & Tate & 3 & 68 & 0.432 \\
\hline 24 & Xilinx xc2vp125 & 56 kSlices & 15 & Tate & 3 & 68 & 0.85 \\
\hline
\end{tabular}


Table 3. Performance and area comparison for scalar multiplication

\begin{tabular}{ccrcccc}
\hline Design & Technology & Area & $\begin{array}{c}\text { Freq. } \\
(\text { MHz })\end{array}$ & $\begin{array}{c}\text { Scalar } \\
\text { Mult. Alg. }\end{array}$ & $\log _{2}\left(\left|\mathbb{F}_{p}\right|\right)$ & $\begin{array}{c}\text { Time } \\
(\text { ms })\end{array}$ \\
\hline this work & $130 \mathrm{~nm}$ std. cell & 97 kGates & 338 & NAF recoding & 256 & 0.998 \\
{$[30$} & $130 \mathrm{~nm}$ std. cell & 122 kGates & 556 & NAF recoding & 256 & 1.01 \\
{$[29$} & $130 \mathrm{~nm}$ std. cell & 107 kGates & 138 & NAF recoding & 256 & 2.68 \\
\hline
\end{tabular}

characteristic give performance figures in terms of time needed for a scalar multiplication with a scalar $k$ of a certain size, i.e. the computation of $[k] P$ for some $P \in E\left(\mathbb{F}_{p}\right)$. An overview is presented in Table 3 .

In order to compare the results of this work with these architectures we implemented scalar multiplication on the 256-bit Barreto-Naehrig curve that we also used for pairing computation. Our design does not accelerate field inversion through hardware, so we use Jacobian projective coordinates to represent the points on the curve, trading inversions for several multiplications.

A scalar multiplication with a 256-bit scalar takes $0.998 \mathrm{~ms}$ for the $128 \mathrm{~m} 2$ variant of the proposed design. This number includes transformation of the scalar into NAF and a transformation from Jacobian into affine coordinates at the end. Note that ASIP variant $128 \mathrm{~m} 2$ is not only slightly faster than the designs in [29] and [30], but also consumes less area.

Application to smart cards. In [10] (updated in [13]), Devigili et al. report $5.17 \mathrm{~s}$ for the computation of the Ate pairing over a 256-bit Barreto-Naehrig curve on a Philips HiPerSmart ${ }^{\mathrm{TM}}$ smart card operating at $20.57 \mathrm{MHz}$. This smart card contains a SmartMIPS-based 32-bit architecture and is manufactured in $180 \mathrm{~nm}$ technology. For interactive processes this execution time is not sufficient even when the smart card operates at its maximum frequency of $38 \mathrm{MHz}$. Our design achieves - synthesized in a $180 \mathrm{~nm}$ CMOS standard cell library with a supply voltage of $1.8 \mathrm{~V}$ - over $230 \mathrm{MHz}$. Even running our smallest design variant 32m1 at the clock speed of $20.57 \mathrm{MHz}$ (leaving a substantial margin for place and route and implementation of protection mechanisms against side channel attacks), the Ate pairing takes $0.71 \mathrm{~s}$ and the Optimal Ate pairing is executed in $0.50 \mathrm{~s}$, which is already sufficient for interactive processes. Depending on the design variant used, speedups of over $20 \times$ could be achieved. This gives an impression of the achievable performance increase for the computation of cryptographic pairings in the embedded domain when more specialized hardware is used.

\section{Conclusion and Outlook}

In this paper we presented a design-space exploration of an ASIP for computation of cryptographic pairings over BN curves. The design is based on extensions of an existing RISC core, which are completely transparent and independent from the original pipeline. Therefore, they could be applied to any RISC-like architecture, 
which can stall the pipeline during multi-cycle operations. The extensions are adaptable in terms of timing and allow for a trade-off between execution time and area. A flexible and transparent memory-architecture extension making use of multiple memories (TIMS) enables fast design space exploration and the usage of existing compilers, since the address space remains unsegmented. We are - up to our knowledge - the first to implement and time a complete implementation of high-security cryptographic pairings on dedicated specialized hardware.

Future objectives include countermeasures against side-channel attacks, which are not implemented in the current design, either in hard- or in software.

\section{References}

1. Menezes, A.J., Okamoto, T., Vanstone, S.A.: Reducing elliptic curve logarithms to logarithms in a finite field. IEEE Trans. Information Theory 39(5), 1639-1646 (1993)

2. Frey, G., Rück, H.G.: A remark concerning $m$-divisibility and the discrete logarithm in the divisor class group of curves. Math. of Computation 62(206), 865-874 (1994)

3. Joux, A.: A one round protocol for tripartite Diffie-Hellman. In: Bosma, W. (ed.) ANTS 2000. LNCS, vol. 1838, pp. 385-394. Springer, Heidelberg (2000)

4. Boneh, D., Franklin, M.: Identity based encryption from the Weil pairing. In: Kilian, J. (ed.) CRYPTO 2001. LNCS, vol. 2139, pp. 213-229. Springer, Heidelberg (2001)

5. Boneh, D., Lynn, B., Shacham, H.: Short signatures from the Weil pairing. J. Cryptology 17(4), 297-319 (2004)

6. Boneh, D.: A brief look at pairings based cryptography. In: Proceedings of the 48th Annual IEEE Symposium on Foundations of Computer Science - FOCS 2007, pp. 19-26 (2007)

7. Barreto, P.S.L.M., Naehrig, M.: Pairing-friendly elliptic curves of prime order. In: Preneel, B., Tavares, S. (eds.) SAC 2005. LNCS, vol. 3897, pp. 319-331. Springer, Heidelberg (2006)

8. Barker, E., Barker, W., Burr, W., Polk, W., Smid, M.: Recommendation for key management - part 1: General (revised). National Institute of Standards and Technology, NIST Special Publication 800-57 (2007)

http://csrc.nist.gov/publications/nistpubs/800-57/ sp800-57-Part1-revised2_Mar08-2007.pdf

9. Näslund, M.: Ecrypt yearly report on algorithms and keysizes (2007-2008) (2008), http://www.ecrypt.eu.org/ecrypt1/documents/D.SPA.28-1.1.pdf

10. Devegili, A.J., Scott, M., Dahab, R.: Implementing cryptographic pairings over Barreto-Naehrig curves. In: Takagi, T., Okamoto, T., Okamoto, E., Okamoto, T. (eds.) Pairing 2007. LNCS, vol. 4575, pp. 197-207. Springer, Heidelberg (2007)

11. Grabher, P., Großschädl, J., Page, D.: On software parallel implementation of cryptographic pairings. Cryptology ePrint Archive, Report 2008/205 (2008), http://eprint.iacr.org/2008/205

12. Naehrig, M., Barreto, P.S.L.M., Schwabe, P.: On compressible pairings and their computation. In: Vaudenay, S. (ed.) AFRICACRYPT 2008. LNCS, vol. 5023, pp. 371-388. Springer, Heidelberg (2008)

13. Devegili, A.J., Scott, M., Dahab, R.: Implementing cryptographic pairings over Barreto-Naehrig curves. Cryptology ePrint Archive, Report 2007/309 (2007), http://eprint.iacr.org/2007/390 
14. Beuchat, J.-L., Brisebarre, N., Detrey, J., Okamoto, E., Rodríguez-Henríquez, F.: A comparison between hardware accelerators for the modified Tate pairing over $\mathbb{F}_{2}$ m and $\mathbb{F}_{3}$. In: Galbraith, S.D., Paterson, K.G. (eds.) Pairing 2008. LNCS, vol. 5209, pp. 297-315. Springer, Heidelberg (2008)

15. Beuchat, J.-L., Brisebarre, N., Detrey, J., Okamoto, E., Shirase, M., Takagi, T.: Algorithms and arithmetic operators for computing the $\eta_{t}$ pairing in characteristic three. IEEE Trans. Comput. 57(11), 1454-1468 (2008)

16. Beuchat, J.-L., Shirase, M., Takagi, T., Okamoto, E.: An algorithm for the $\eta_{t}$ pairing calculation in characteristic three and its hardware implementation. In: Proc. 18th IEEE Symp. Computer Arithmetic - ARITH 2007, pp. 97-104 (2007)

17. Beuchat, J.-L., Doi, H., Fujita, K., Inomata, A., Kanaoka, A., Katouno, M., Mambo, M., Okamoto, E., Okamoto, T., Shiga, T., Shirase, M., Soga, R., Takagi, T., Vithanage, A., Yamamoto, H.: FPGA and ASIC implementations of the $\eta_{t}$ pairing in characteristic three. Cryptology ePrint Archive, Report 2008/280 (2008), http://eprint.iacr.org/2008/280

18. Shu, C., Kwon, S., Gaj, K.: FPGA accelerated Tate pairing based cryptosystems over binary fields. In: Proc. IEEE Int'l Conf. Field Programmable Technology FPT 2006, pp. 173-180 (2006)

19. Keller, M., Ronan, R., Marnane, W., Murphy, C.: Hardware architectures for the Tate pairing over GF $\left(2^{m}\right)$. Computers \& Electrical Eng. 33(5-6), 392-406 (2007)

20. Keller, M., Kerins, T., Crowe, F., Marnane, W.: FPGA implementation of a GF $\left(2^{m}\right)$ Tate pairing architecture. In: Bertels, K., Cardoso, J.M.P., Vassiliadis, S. (eds.) ARC 2006. LNCS, vol. 3985, pp. 358-369. Springer, Heidelberg (2006)

21. Ronan, R., Ó hÉigeartaigh, C., Murphy, C., Scott, M., Kerins, T.: FPGA acceleration of the Tate pairing in characteristic 2. In: Proc. IEEE Int'l Conf. Field Programmable Technology, pp. 213-220 (2006)

22. Grabher, P., Page, D.: Hardware acceleration of the Tate pairing in characteristic three. In: Rao, J.R., Sunar, B. (eds.) CHES 2005. LNCS, vol. 3659, pp. 398-411. Springer, Heidelberg (2005)

23. Jiang, J.: Bilinear pairing (Eta_T pairing) IP core. Technical report (2007), http://www.cs.cityu.edu.hk/ ecc/doc/etat_datasheet_v2.pdf

24. Kerins, T., Marnane, W.P., Popovici, E.M., Barreto, P.S.L.M.: Efficient hardware for the Tate pairing calculation in characteristic three. In: Rao, J.R., Sunar, B. (eds.) CHES 2005. LNCS, vol. 3659, pp. 412-426. Springer, Heidelberg (2005)

25. Ronan, R., Murphy, C., Kerins, T., Ó hÉigeartaigh, C., Barreto, P.S.L.M.: A flexible processor for the characteristic $3 \eta_{t}$ pairing. Int'l J. High Performance Systems Architecture 1(2), 79-88 (2007)

26. Kömürcü, G., Savas, E.: An efficient hardware implementation of the Tate pairing in characteristic three. In: Proc. Third Int'l Conf. Systems - ICONS 2008, pp. $23-28$ (2008)

27. Barenghi, A., Bertoni, G., Breveglieri, L., Pelosi, G.: A FPGA coprocessor for the cryptographic Tate pairing over $\mathbb{F}_{p}$. In: Proc. Fifth Int'l Conf. Information Technology: New Generations - ITNG 2008, pp. 112-119 (2008)

28. Vejda, T., Page, D., Großschädl, J.: Instruction set extensions for pairing-based cryptography. In: Takagi, T., Okamoto, T., Okamoto, E., Okamoto, T. (eds.) Pairing 2007. LNCS, vol. 4575, pp. 208-224. Springer, Heidelberg (2007)

29. Satoh, A., Takano, K.: A scalable dual-field elliptic curve cryptographic processor. IEEE Trans. Computers 52(4), 449-460 (2003)

30. Chen, G., Bai, G., Chen, H.: A high-performance elliptic curve cryptographic processor for general curves over $\mathrm{GF}(\mathrm{p})$ based on a systolic arithmetic unit. IEEE Trans. Circuits and Systems II: Express Briefs 54(5), 412-416 (2007) 
31. Güneysu, T., Paar, C.: Ultra high performance ECC over NIST primes on commercial FPGAs. In: Oswald, E., Rohatgi, P. (eds.) CHES 2008. LNCS, vol. 5154, pp. 62-78. Springer, Heidelberg (2008)

32. Galbraith, S.: Pairings. In: Blake, I.F., Seroussi, G., Smart, N.P. (eds.) Advances in Elliptic Curve Cryptography. London Mathematical Society Lecture Note Series, Cambridge University Press, Cambridge (2005)

33. Miller, V.S.: The Weil pairing, and its efficient calculation. J. Cryptology 17, 235$261(2004)$

34. Hess, F., Smart, N.P., Vercauteren, F.: The Eta pairing revisited. IEEE Trans. Information Theory 52(10), 4595-4602 (2006)

35. Lee, E., Lee, H.S., Park, C.M.: Efficient and generalized pairing computation on Abelian varieties. Cryptology ePrint Archive, Report 2008/040 (2008), http://eprint.iacr.org/2008/040

36. Vercauteren, F.: Optimal pairings. Cryptology ePrint Archive, Report 2008/096 (2008), http://eprint.iacr.org/2008/096

37. Hess, F.: Pairing lattices. In: Galbraith, S.D., Paterson, K.G. (eds.) Pairing 2008. LNCS, vol. 5209, pp. 18-38. Springer, Heidelberg (2008)

38. Barreto, P.S.L.M., Galbraith, S.D., Ó hÉigeartaigh, C., Scott, M.: Efficient pairing computation on supersingular Abelian varieties. Designs, Codes and Cryptography 42(3), 239-271 (2007)

39. Barreto, P.S.L.M., Kim, H.Y., Lynn, B., Scott, M.: Efficient algorithms for pairingbased cryptosystems. In: Yung, M. (ed.) CRYPTO 2002. LNCS, vol. 2442, pp. 354-368. Springer, Heidelberg (2002)

40. CoWare: Processor Designer (2009), http://www. coware.com/products/processordesigner.php

41. National Institute of Standards and Technology, NIST: FIPS 186-2: Digital Signature Standard (DSS) (2000), http://csrc.nist.gov/publications/fips/ fips186-2/fips186-2-change1.pdf

42. Bernstein, D.J.: Curve25519: new Diffie-Hellman speed records. In: Yung, M., Dodis, Y., Kiayias, A., Malkin, T.G. (eds.) PKC 2006. LNCS, vol. 3958, pp. $207-$ 228. Springer, Heidelberg (2006)

43. Montgomery, P.: Modular multiplication without trial division. Mathematics of Computation 44(170), 519-521 (1985)

44. Nibouche, O., Bouridane, A., Nibouche, M.: Architectures for Montgomery's multiplication. IEE Proc. - Computers and Digital Techniques 150(6), 361-368 (2003)

45. Synopsys: Design Compiler (2009), http://www.synopsys.com/products/logic/design_compiler.html

46. Shu, C., Kwon, S., Gaj, K.: FPGA accelerated Tate pairing based cryptosystems over binary fields. Cryptology ePrint Archive, Report 2006/179 (2006), http://eprint.iacr.org/2006/179 